\title{
Adipose stem cells from patients with Crohn's disease show a distinctive DNA methylation pattern
}

\author{
Carolina Serena ${ }^{1,2}$, Monica Millan ${ }^{3,4}$, Miriam Ejarque ${ }^{1,2}$, Alfonso Saera-Vila1', Elsa Maymó-Masip 1,2,
} Catalina Núñez-Roa ${ }^{1,2}$, Diandra Monfort-Ferré ${ }^{1}$, Margarida Terrón-Puigg ${ }^{1,2}$, Michelle Bautista ${ }^{5}$, Margarita Menacho ${ }^{5}$, Marc Martí ${ }^{6}$, Eloy Espin ${ }^{6}$, Joan Vendrell ${ }^{1,2,7^{*}}$ and Sonia Fernández-Veledo ${ }^{1,2^{*}}$

\begin{abstract}
Background: Crohn's disease (CD) is characterized by persistent inflammation and ulceration of the small or large bowel, and expansion of mesenteric adipose tissue, termed creeping fat (CF). We previously demonstrated that human adipose-derived stem cells (hASCs) from CF of patients with CD exhibit dysfunctional phenotypes, including a pro-inflammatory profile, high phagocytic capacity, and weak immunosuppressive properties. Importantly, these phenotypes persist in patients in remission and are found in all adipose depots explored including subcutaneous fat. We hypothesized that changes in hASCs are a consequence of epigenetic modifications.
\end{abstract}

Methods: We applied epigenome-wide profiling with a methylation array (Illumina EPIC/850k array) and gene expression analysis to explore the impact of CD on the methylation signature of hASCs isolated from the subcutaneous fat of patients with CD and healthy controls ( $n=7$ and 5 , respectively; cohort I). Differentially methylated positions ( $p$ value cutoff $<1 \times 10^{-4}$ and ten or more DMPs per gene) and regions (inclusion threshold $0.2, p$ value cutoff $<1 \times 10^{-2}$ and more than 2 DMRs per gene) were identified using dmpfinder and Bumphunter (minfi), respectively. Changes in the expression of differentially methylated genes in hASCs were validated in a second cohort $(n=10 / 10$ inactive and active CD and 10 controls; including patients from cohort I) and also in peripheral blood mononuclear cells (PBMCs) of patients with active/inactive CD and of healthy controls (cohort III; $n=30$ independent subjects).

Results: We found a distinct DNA methylation landscape in hASCs from patients with CD, leading to changes in the expression of differentially methylated genes involved in immune response, metabolic, cell differentiation, and development processes. Notably, the expression of several of these genes in hASCs and PBMCs such as tumor necrosis factor alpha (TNFA) and PR domain zinc finger protein 16 (PRDM16) were not restored to normal (healthy) levels after disease remission.

Conclusions: hASCs of patients with CD exhibit a unique DNA methylation and gene expression profile, but the expression of several genes are only partially restored in patients with inactive CD, both in hASCs and PBMCs. Understanding how CD shapes the functionality of hASCs is critical for investigating the complex pathophysiology of this disease, as well as for the success of cell-based therapies.

Keywords: Methylome, Inflammatory bowel disease, Adipose tissue, Epigenetics, Gene expression

\footnotetext{
*Correspondence: jvo@comt.es; sonia.fernandezveledo@gmail.com

'Institut d'Investigació Sanitària Pere Virgili, Hospital Universitari Joan XXIII, Dr Mallafré Guasch, 4, 43007 Tarragona, Spain

Full list of author information is available at the end of the article
}

\section{$\triangle B M C$}

(C) The Author(s). 2020 Open Access This article is licensed under a Creative Commons Attribution 4.0 International License, which permits use, sharing, adaptation, distribution and reproduction in any medium or format, as long as you give appropriate credit to the original author(s) and the source, provide a link to the Creative Commons licence, and indicate if changes were made. The images or other third party material in this article are included in the article's Creative Commons licence, unless indicated otherwise in a credit line to the material. If material is not included in the article's Creative Commons licence and your intended use is not permitted by statutory regulation or exceeds the permitted use, you will need to obtain permission directly from the copyright holder. To view a copy of this licence, visit http://creativecommons.org/licenses/by/4.0/ The Creative Commons Public Domain Dedication waiver (http://creativecommons.org/publicdomain/zero/1.0/) applies to the data made available in this article, unless otherwise stated in a credit line to the data. 


\section{Background}

The prevalence of inflammatory bowel disease (IBD), including Crohn's disease (CD) and ulcerative colitis, is increasing, especially in western countries, and it is likely to represent an important social and economic burden in the coming years $[1,2]$. CD is characterized by persistent inflammation and ulcerations at the small or large bowel, provoking chronic low-grade systemic inflammation and an undulating course of activity, with relapsing cycles occurring after periods of remission. Adipose tissue (AT) is believed to play an active role in the pathogenesis of $\mathrm{CD}$, as the expansion of mesenteric fat attached to the inflamed segments of intestine, also known as "creeping fat," is a hallmark of the disease that seems to be directly related to disease activity [3-6]. In fact, it has recently been demonstrated that inclusion of the mesentery in ileocolic resection reduces the rate of surgical recurrence [7].

AT is now recognized as a highly active endocrine organ that controls metabolic homeostasis. Emerging evidence points to the immune system as a major contributor to AT homeostasis and function [8]. Indeed, AT contributes importantly to low-grade systemic inflammation in obesity and its comorbidities [9-11]. AT contains an abundant population of multipotent human adiposederived stem cells (hASCs) that differentiate into several specialized cells, including adipocytes and endothelial cells, and play key roles in immune response [12, 13]. For example, hASCs administered to the circulation have the ability to home to inflamed/injured tissues [14, 15]. Of note, some metabolic stress-induced conditions including $\mathrm{CD}$ alter the anti-inflammatory properties of these cells [16-18]. Remarkably, the altered properties of hASCs persist even when patients with CD are in remission, in all AT depots explored including subcutaneous fat [18]. The constancy of this dysfunctional phenotype might reflect changes at the epigenetic level, which ultimately hamper the resolution of the inflammatory events in the sinus of the affected mesenteric AT. In support of this proposition, recent DNA methylation studies in peripheral blood mononuclear cells (PBMCs) revealed that patients with $\mathrm{CD}$ have a distinct DNA methylome linked to the expression of differentially methylated genes associated with immune response and inflammation [1921]. We hypothesized that hASCs from patients with CD are conditioned by a chronic inflammatory milieu, which may alter their DNA methylome and influence their antiinflammatory and regenerative capacity.

To address this issue, we designed a three-step study: first, we evaluated whether hASCs from the subcutaneous fat of patients with $\mathrm{CD}$ have a unique methylation signature that would alter their gene expression profile; second, in a confirmatory study, we performed gene expression analysis in patients considering disease activity; and third, we assessed the reproducibility of the changes in gene expression of candidates genes in an more easily accessible sample, by testing for a specific blood signature.

\section{Results \\ Methylation signature of adipose stem cells from subcutaneous fat in patients with Crohn's}

We first assessed genome-wide patterns of DNA methylation in hASCs isolated from subcutaneous fat depots of patients with CD and compared them with those from hASCs isolated from healthy controls $(n=7$ patients with $\mathrm{CD}$ and 5 healthy controls; cohort I). No differences in overall mean DNA methylation (calculated by averaging across all probes on the Illumina EPIC/850k array) were observed between the two groups ( $p=0.33$; Student's $t$ test) (data not shown), indicating that CD is not associated with global changes to DNA methylation in this cell type. By contrast, DNA methylation at individual $\mathrm{CpG}$ sites showed considerable variability between $\mathrm{CD}$ and controls. Principal component analysis (PCA) of the first two principal components (PC1 and $\mathrm{PC} 2$ ) of the methylation data revealed a difference between hASCs of CD and those of controls (Fig. 1a) with PC1 explaining $20.56 \%$ of the variance. We also performed a correlation analysis between the confounding variables, hospital, sex, age, and body mass index (BMI) and the principal components (Fig. 1B; data is represented by a heat map). No correlation was found between the six principal components and hospital, sex, age, or BMI, suggesting that these confounding variables did not significantly affect the methylome pattern. Notably, we found a significant correlation between the presence or absence of the disease and PC1 (Fig. 1b).

It is now recognized that, in addition to the expected binary behavior of $\mathrm{CpG}$ methylation (either hypo- or hypermethylated), some cytosines show intermediate, yet low, levels of methylation [22]. Thus, we explored potential differences in the segmented methylomes as fully methylated regions (FMRs; >50\% methylated CpGs), low-methylated regions (LMRs; 3.9-50\%), and unmethylated regions (UMRs; $1-3.9 \%$ ), as described [22-26]. The FMR class comprised over $50 \%$ of the methylome in all samples (Fig. 1c), and the global percentage of methylation was stable between control- and CD-hASCs. Nevertheless, there was a trend for greater methylation in the LMR region in hASCs isolated from patients with $C D$ relative to those from healthy controls.

\section{Differentially methylated positions in adipose stem cells isolated from patients with Crohn's disease}

We found statistically significant differences in 18,253 CpGs between CD- and control-hASCs. To specifically evaluate the methylation patterns and the preferential genome location, we segmented all significant differentially 


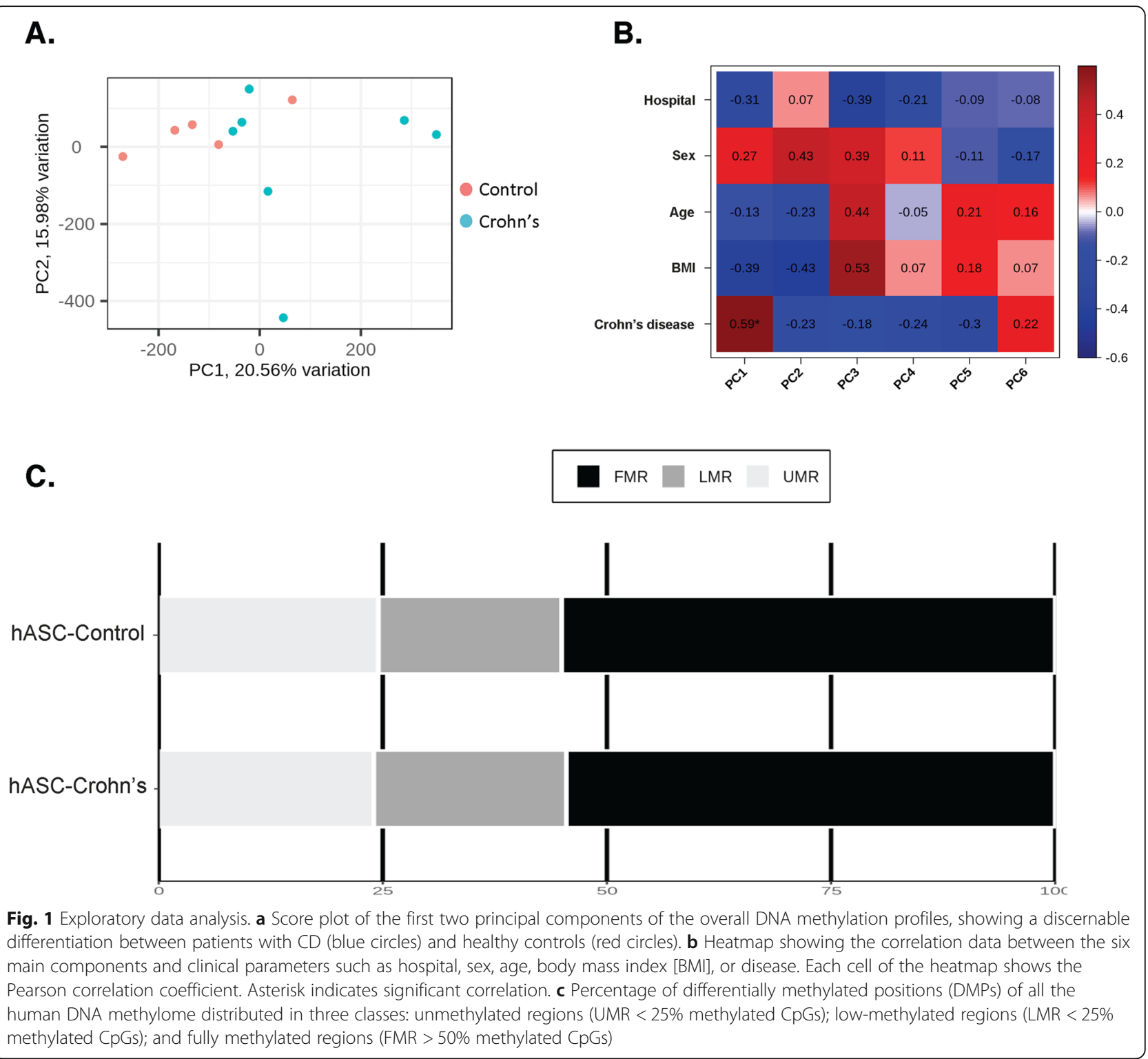

methylated positions (DMPs) with a $p$ value $<1 \times$ $10^{-4}$ into the following eight categories according to the Illumina annotations: transcription start site (TSS) 1500, TSS 200 (indicating the number of bases upstream), 5' untranslated region (UTR), 1st exon, ExonBnd (exon boundary), gene body, 3' UTR, and no gene location (Fig. 2a, left panel). The DMP distribution within a CpG island is shown in Fig. 2a (right panel), with the majority located in the open sea region (>50\%). We found that $35 \%$ of methylation sites occurred in the promoter region (including TSS 1500, TSS 200, and $5^{\prime}$ UTR), which is related to gene silencing [23, 27] (Fig. 2b). Of note, most of the methylation sites were found in the gene body (45\%) (Fig. 2b), which is known to have a positive correlation with gene transcription [23, 28].
We next performed a heatmap analysis of the significant DMPs between the two groups, which revealed that the DMPs segregated into two clusters (Fig. 2c). We performed a second PCA using only the significant DMPs between hASCs from CD and controls, finding that the explained variance increased considerably when compared with the first PCA (Fig. 2d), with PC1 accounting for $85.3 \%$ of the variance. Additionally, we generated a Manhattan plot to observe, at the chromosomal level, where the significant DMPs were located. The results of this analysis indicated that all of the DMPs were evenly distributed throughout the chromosomal complement (Fig. 2e).

Finally, we assigned DMPs found in hASCs between CD and control individuals to candidate genes, which were identified based on a nominal $p$ value cutoff of $<1 \times 10^{-4}$ 
A.

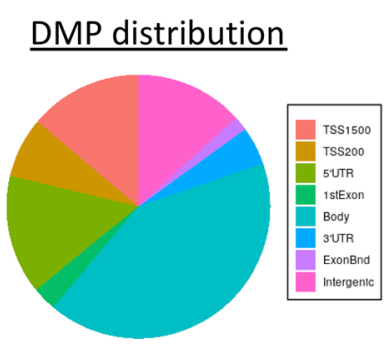

$\underline{\mathrm{CpG} \text { allocation }}$

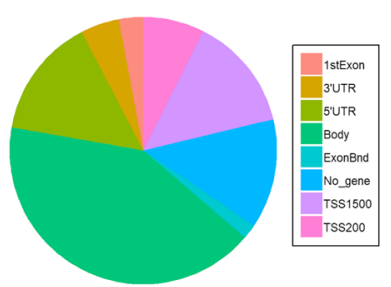

c.
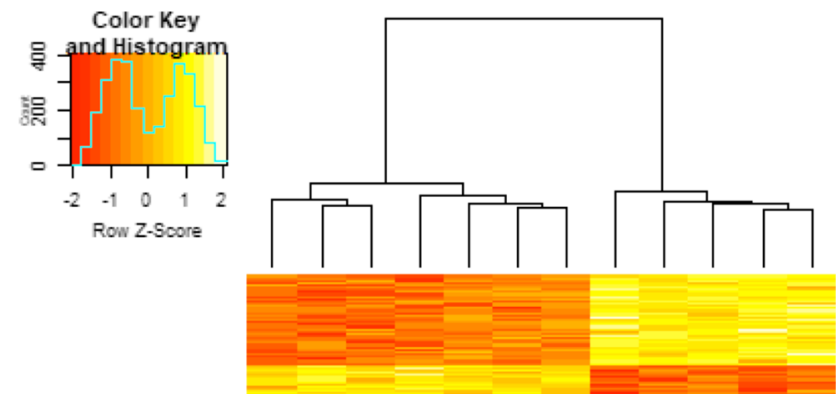

B.

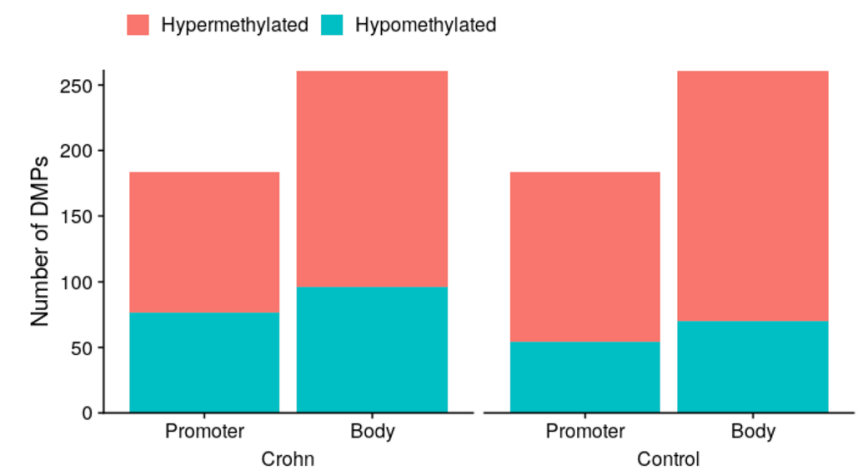

D.

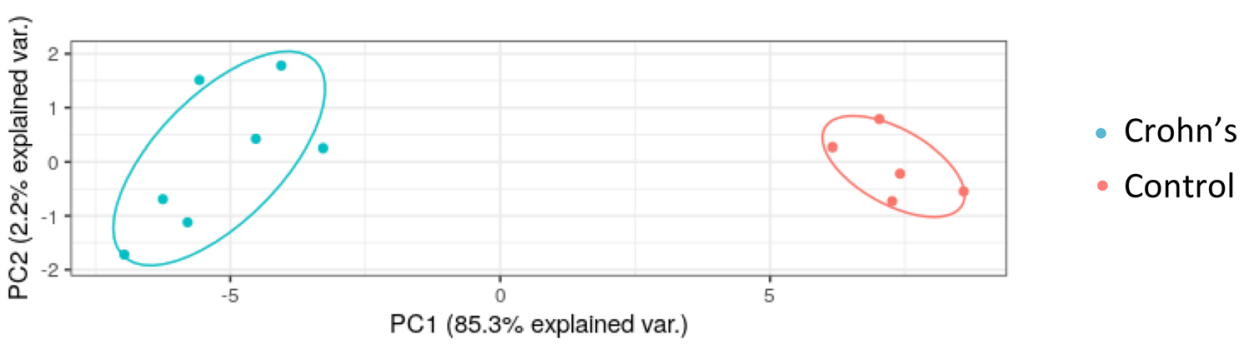

E.

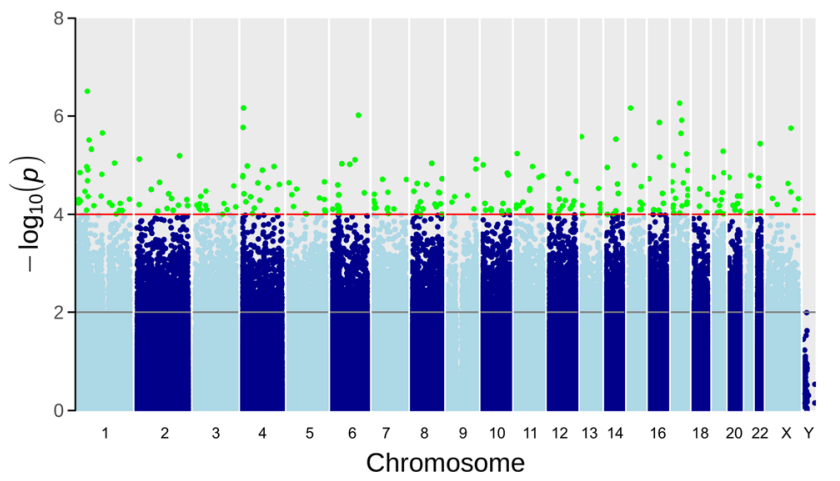

F.

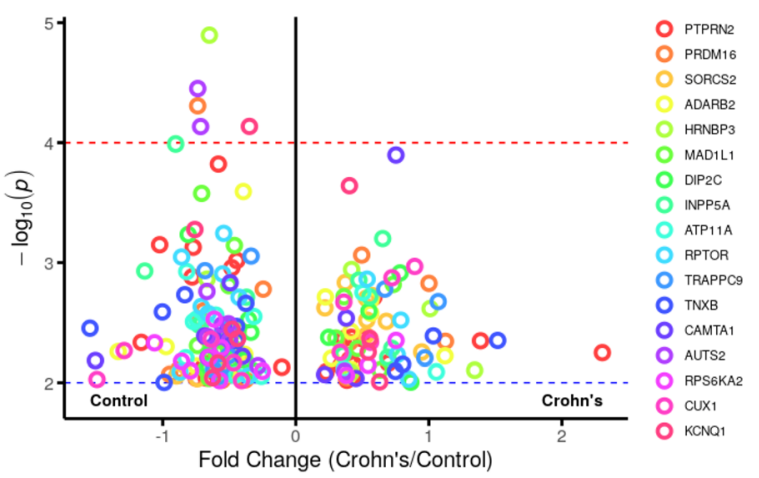

Fig. 2 (See legend on next page.) 
(See figure on previous page.)

Fig. 2 Differentially methylated position analysis in adipose-derived stem cells isolated from patients with Crohn's disease and healthy controls. a Intragenic differentially methylated position (DMP) distribution: approximately $45 \%$ of the DMPs are located in the gene body and $35 \%$ are located in the promoter. CpG allocation: > 50\% of the CpGs are in the open sea region. b Distribution of hypo- and hypermethylated DMPs for Crohn's-hASC and control-hASCs. c Heatmap and hierarchical clustering of CpGs according to their methylation profile in Crohn's disease patients compared with healthy donors. Red bars represent hypomethylated positions and yellow bars hypermethylated positions. $\mathbf{d}$ Principal component analysis demonstrating the first two main components of the significant CpG data set. e Manhattan plot of all DMPs. Significant DMPs are colored in green, achieving the significance threshold $(p<0.0001)$. $\mathbf{f}$ Volcano plot showing the top DMPs associated with genes. Visualization of the genes with the highest number of significant CpGs. Negative values (points to the left of the graph) indicate hypomethylated; positive values (points to the right of the graph) indicate hypermethylated. ExonBnd, exon boundaries; UTR, untranslated region; TSS, transcription start site; hASCs, human adipose-stem cells

and ten or more DMPs per gene (Supplementary File 1). As illustrated in the volcano plot in Fig. $2 \mathrm{f}, 17$ genes showed significant changes in DMPs: PTPRN2 (with 28 significant CpGs); PRDM16 (with 17 significant CpGs); SORC2, ADARB2, and HRNBP3 (with 16 significant CpGs); MAD1L1 (with 14 significant CpGs); DIPC2 and INPP5A (with 13 significant CpGs), ATP11A, and RPTOR (with 12 significant CpGs); TRAPPC9, TNBX, CAMTA1, AUST2, and RPS6KA2 (with 11 significant CpGs); and CUX1 and KCNQ1 (with 10 significant CpGs).

\section{Differentially methylated regions in hASCs isolated from patients with Crohn's disease}

Differentially methylated regions (DMRs) were located using the minfi function Bumphunter, using default settings and the smoothing option. Bump hunting with smoothing is a useful methodology for finding regions of biological interest in the context of DNA methylation studies [29]. From our DMR analysis, we found 2150 regions located near known annotated genes (Supplementary File 2). Including those genes with two or more significant DMRs and a $p$ value cutoff of $<1 \times 10^{-2}$, we obtained 158 regions annotated with known genes. From these, we specifically selected two or more significant DMRs annotated with the same gene that showed the same direction of effect and were located in the promoter or body regions. We discriminated 37 and 35 genes that we defined as up- or downregulated, respectively, in hASCs of patients with CD. To address the molecular and functional pathways implicated for these genes, we performed functional analysis using STRING v.11.00 (https://string-db.org/). Interestingly, the network analysis of upregulated genes in CD-hASCs revealed a significant protein-protein interaction (PPI) enrichment of the network with a $p$ value of $1.79 \times 10^{-7}$, indicating that the proteins were at least partially biologically connected as a group. We selected the more enriched pathways, which formed three categories: immune system, metabolic process, and cell differentiation (Fig. 3a and Table 1). Immune system included 21 enriched gene ontology (GO) terms with a false discovery rate (FDR) ranging from 0.0229 to 0.0484 ; metabolic process included 7 enriched GO terms with an FDR ranging from 0.0229 to
0.0499; and cell differentiation included 5 enriched GO terms with an FDR ranging from 0.0229 to 0.0358 . These categories were confirmed using REVIGO (http://revigo. irb.hr/), which summarizes and visualizes lists of GO terms (Supplementary Figure S1). Of note, REVIGO highlighted immune system process as the most important category affecting hASCs of patients with CD.

The network analysis of downregulated genes in CDhASCs revealed a significant PPI enrichment of the network with a $p$ value of $4.44 \times 10^{-4}$ (Fig. $3 \mathrm{~b}$ ). The more enriched pathways were development process (included 4 enriched GO terms with an FDR ranging from 0.0209 to 0.0374 ) and cell differentiation (included 1 enriched GO term with an FDR of 0.0374) (Table 1), which were confirmed using REVIGO (Supplementary Figure S2). The most enriched GO terms of each category as well as the related genes are described in Table 1.

\section{Confirmatory gene expression analysis in patients with Crohn's disease with regard to disease activity status}

Because DNA methylation can affect transcription, we examined the mRNA expression of a representative selection of at least 3-4 genes per category of identified genes both in patients, according to $\mathrm{CD}$ activity, and in healthy subjects, using samples from cohort II (Table 1). To confirm CD activity, we calculated the Crohn's disease activity index (CDAI) score and assessed clinical and biological parameters that included PCR and fecal calprotectin determinations. Also, an endoscopy evaluation was performed in $80 \%$ of the patients (Supplementary Table S2).

\section{Immune system}

The expression of immune system-related genes such as complement $2(C 2)$, inhibitor of nuclear factor kappa B kinase subunit epsilon (IKBKE), inhibitor of nuclear factor kappa B kinase subunit beta $(I K B K B)$, lymphotoxin beta receptor $(L T B R)$, spondin 2 (SPON2), chitinase domaincontaining 1 (CHID1), and tumor necrosis factor alpha (TNFA) was significantly higher in hASCs isolated from patients with active disease than in equivalent cells from controls (Fig. 4a). The results were in agreement with the methylation data, and we found significant correlations between gene expression and methylation in some of 
A.

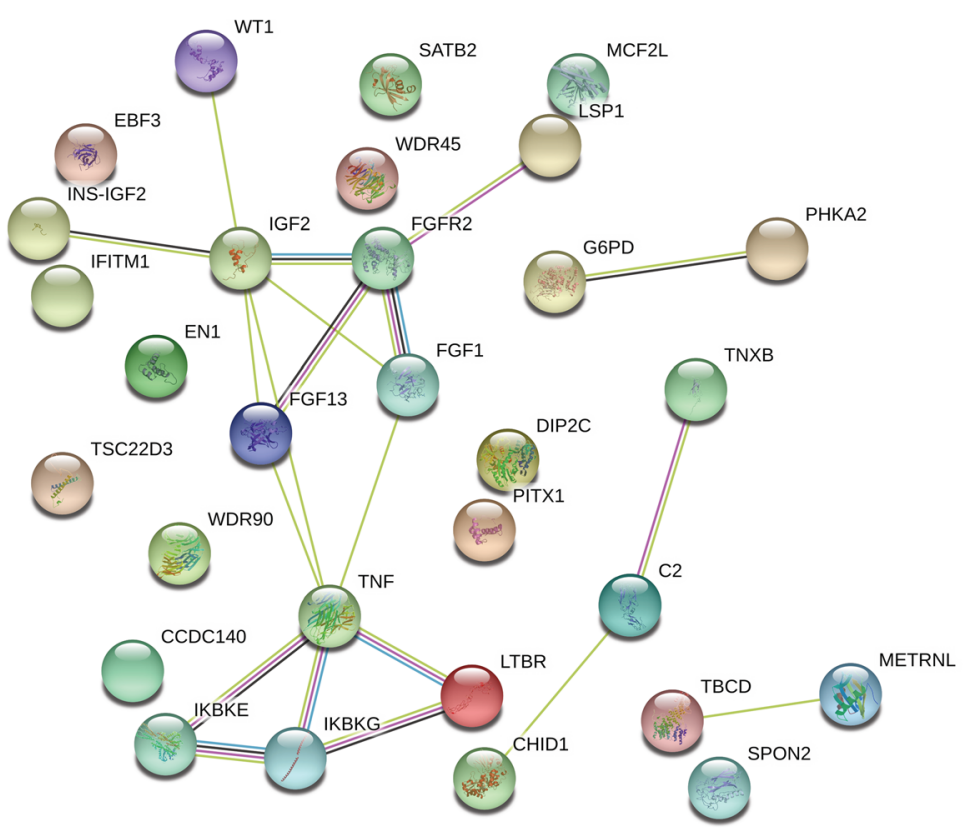

Interactions of proteins performed with STRING database up regulated in adipose -stem cells of Crohn's disease patients.

number of nodes: 30

average node degree: 1.33

avg. local clustering coefficient: 0.462

number of edges: 20

expected number of edges: 5

PPI enrichment $p$-value: $1.79 \mathrm{e}-07$

B.

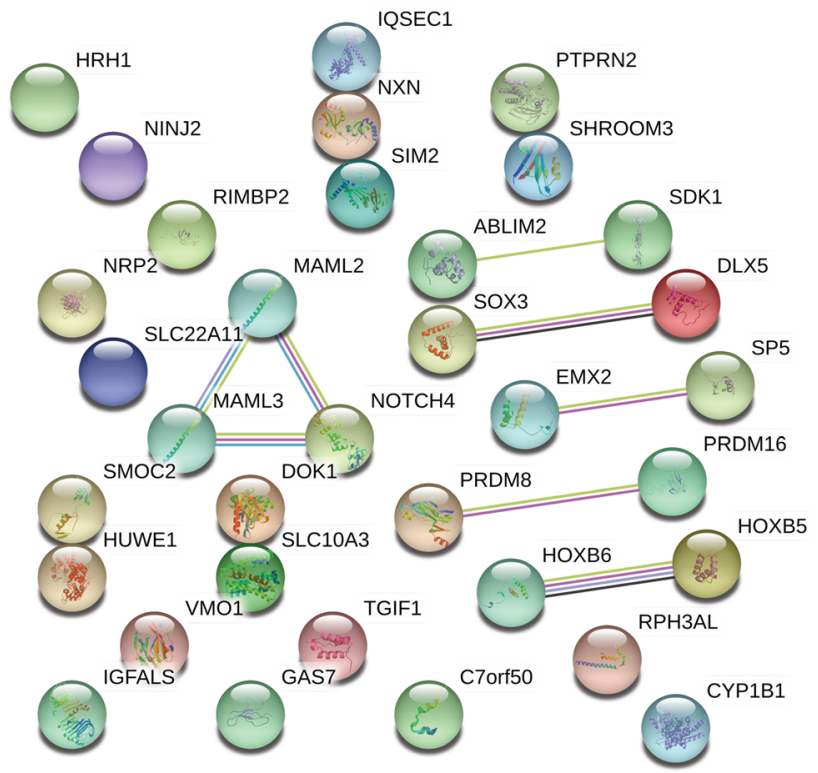

Interactions of proteins performed with STRING database down regulated in adipose-stem cells of Crohn's disease patients.

number of nodes: 34

number of edges: 8

average node degree: 0.471

expected number of edges: 2

avg. local clustering coefficient: 0.382

PPI enrichment $p$-value: 4.44e-04

Fig. 3 Functional analysis of gene-associated differentially methylated regions in Crohn's disease. a Network of the 30 genes upregulated in Crohn's disease. b Network of the 34 genes downregulated in Crohn's disease. Such enrichment indicates that the proteins are at least partially biologically connected as a group 
Table 1 Significantly enriched GO terms of biological processes for genes identified as up- or downregulated in hASCs from patients with Crohn's disease

\begin{tabular}{|c|c|c|c|}
\hline GO Term & Biological Process & False Discovery Rate & Marker Gene \\
\hline \multicolumn{4}{|c|}{ UP REGULATED IN CROHN-hASCS } \\
\hline \multicolumn{4}{|c|}{ Immune system } \\
\hline GO:0002682 & regulation of immune system process & 0.0229 & C2,IFITM1,IGF2,IKBKE,IKBKG,SPON2,TNF,TSC22D3 \\
\hline GO:0006952 & defense response & 0.0229 & C2,CHID1,IFITM1,IKBKE,IKBKG,LSP1,LTBR,SPON2,TNF \\
\hline GO:0007249 & I-kappaB kinase/NF-kappaB signaling & 0.0229 & IKBKE,IKBKG,TNF \\
\hline GO:0050727 & regulation of inflammatory response & 0.0229 & C2,CHID1,METRNL,TNF \\
\hline GO:0009615 & response to virus & 0.0229 & IFITM1,|KBKE,|KBKG,SPON2,TNF \\
\hline GO:0032496 & response to lipopolysaccharide & 0.0229 & FGFR2,LTBR,SPON2,TNF \\
\hline GO:0035456 & response to interferon-beta & 0.0229 & IFITM1,|KBKE \\
\hline GO:0043123 & positive regulation of I-kappaB kinase/NF-kappaB signaling & 0.0229 & IKBKE,IKBKG,LTBR,TNF \\
\hline G0:0045087 & innate immune response & 0.0229 & C2,CHID1,IFITM1,IKBKE,IKBKG,SPON2 \\
\hline GO:0051707 & response to other organism & 0.0229 & FGFR2,IFITM1,IKBKE,IKBKG,LTBR,SPON2,TNF \\
\hline G0:0050776 & regulation of immune response & 0.024 & C2,IFITM1,IKBKE,IKBKG,SPON2,TNF \\
\hline GO:0050778 & positive regulation of immune response & 0.0241 & C2,IKBKE,IKBKG,SPON2,TNF \\
\hline GO:0006955 & immune response & 0.0244 & C2,CHID1,IFITM1,IKBKE,IKBKG,LTBR,SPON2,TNF \\
\hline GO:0002684 & positive regulation of immune system process & 0.0245 & C2,IGF2,IKBKE,IKBKG,SPON2,TNF \\
\hline GO:0002376 & immune system process & 0.0252 & C2,CHID1,FGFR2,G6PD,IFITM1,IKBKE,IKBKG,LTBR,SPON2,TNF \\
\hline G0:0050766 & positive regulation of phagocytosis & 0.0282 & $\mathrm{C} 2, \mathrm{TNF}$ \\
\hline GO:0010803 & regulation of tumor necrosis factor-mediated signaling pathway & 0.0339 & IKBKG,TNF \\
\hline GO:0002718 & regulation of cytokine production involved in immune response & 0.0453 & SPON2,TNF \\
\hline GO:0002920 & regulation of humoral immune response & 0.0457 & C2,TNF \\
\hline GO:0034340 & response to type I interferon & 0.0457 & IFITM1,IKBKE \\
\hline GO:0032755 & positive regulation of interleukin- 6 production & 0.0484 & SPON2,TNF \\
\hline \multicolumn{4}{|c|}{ Metabolic process } \\
\hline G0:0006006 & glucose metabolic process & 0.0229 & G6PD,IGF2,TNF \\
\hline GO:0005975 & carbohydrate metabolic process & 0.0229 & CHID1,G6PD,IGF2,PHKA2,TNF \\
\hline 60:0010604 & positive regulation of macromolecule metabolic process & 0.0229 & EBF3,EN1,FGF1,FGF13,FGFR2,IGF2,IKBKG,LTBR,PITX1,SATB2,TNF,WT1 \\
\hline GO:0031325 & positive regulation of cellular metabolic process & 0.0229 & EBF3,EN1,FGF1,FGF13,FGFR2,IGF2,IKBKG,LTBR,PITX1,SATB2,TNF,WT1 \\
\hline G0:0051173 & positive regulation of nitrogen compound metabolic process & 0.0229 & EBF3,EN1,FGF1,FGF13,FGFR2,IGF2,IKBKG,LTBR,PITX1,SATB2,TNF,WT1 \\
\hline GO:0062013 & positive regulation of small molecule metabolic process & 0.0229 & FGF1,IGF2,TNF \\
\hline 60:0051172 & negative regulation of nitrogen compound metabolic process & 0.0499 & EN1,FGFR2,G6PD,IGF2,PITX1,SATB2,TNF,TSC22D3,WT1 \\
\hline \multicolumn{4}{|c|}{ Cell Differentiation } \\
\hline GO:0030154 & cell differentiation & 0.0229 & EN1,FGF1,FGF13,FGFR2,G6PD,IGF2,LTBR,METRNL,PITX1,SATB2,SPON2,TBCD,TNF,WT1 \\
\hline 60:0001503 & ossification & 0.0229 & FGFR2,IFITM1,IGF2,SATB2 \\
\hline G0:0045667 & regulation of osteoblast differentiation & 0.0229 & FGFR2,IFITM1,TNF \\
\hline G0:0051147 & regulation of muscle cell differentiation & 0.0229 & FGFR2,G6PD,IGF2 \\
\hline G0:0030099 & myeloid cell differentiation & 0.0358 & G6PD,LTBR,TNF \\
\hline \multicolumn{4}{|c|}{ DOWN REGULATED IN CROHN-hASCS } \\
\hline \multicolumn{4}{|c|}{ Development processes } \\
\hline 60:0048731 & system development & 0.0209 & CYP1B1,DLX5,DOK1,EMX2,GAS7,HOXB5,HOXB6,NINJ2,NOTCH4,NRP2,NXN,PRDM16,PRDM8,SDK1,SHROOM33,SIM2,SOX3,SP5 \\
\hline 60:0007275 & multicellular organism development & 0.0218 & CYP1B1,DLX5,DOK1,EMX2,GAS7,HOXB5,HOXB6,NINJ2,NOTCH4,NRP2,NXN,PRDM16,PRDM8,SDK1,SHROOM3,SIM2,SOX3,SP5,TGIF1 \\
\hline 60:0032502 & developmental process & 0.0299 & CYP1B1,DLX5,DOK1,EMX2,GAS7,HOXB5,HOXB6,HUWE1,NINJ2,NOTCH4,NRP2,NXN,PRDM16,PRDM8,SDK1,SHROOM3,SIM2,SOX3,SP5,TGIF1 \\
\hline G0:0007399 & nervous system development & 0.0374 & DLX5,DOK1,EMX2,GAS7,NINJ2,NRP2,PRDM16,PRDM8,SDK1,SHROOM3,SIM2,SOX3 \\
\hline \multicolumn{4}{|c|}{ Cell Diferentiation } \\
\hline G0:0030154 & cell differentiation & 0.0374 & DLX5,DOK1,EMX2,GAS7,HOXB5,HUWE1,NOTCH4,NRP2,NXN,PRDM16,PRDM8,SDK1,SHROOM3,SIM2,SOX3 \\
\hline
\end{tabular}

The GO analysis was performed in the STRING database website (http://www. string-db.org)

these genes (Supplementary Figure S3). We observed that the expression of almost all of the genes tested was normalized to control levels in hASCs isolated from patients with inactive disease, indicating that immune system genes affected by methylation marks were for the most part partially restored in patients during episodes of remission. The exception to this was TNFA, whose expression remained high in patients during remission, which is in agreement with previous data [18].

\section{Metabolic process}

Gene expression analysis of metabolic process-related genes is shown in Fig. 4b. Glucose-6-phosphate dehydrogenase $(G 6 P D)$, receptor interacting serine/threonine kinase 1 (RIPK1), fibroblast growth factor 13 (FGF13), and early $\mathrm{B}$ cell factor 3 (EBF3) were upregulated in hASCs of patients with active CD relative to controls, as anticipated by the methylation data. Of note, the expression of these genes remained high in hASCs from patients with inactive disease. This indicates that metabolic changes are maintained in periods of remission and suggests that the global inflammatory environment during disease activity shapes the methylome signature in these cells to maintain a high metabolic activity independently of the clinical status of the patient, as we alluded to in an earlier study [18].

\section{Cell differentiation process}

Gene expression analysis of cell differentiation-related genes is shown in Fig. 4C. Genes upregulated in hASCs from patients with active disease relative to controls included engrailed homeobox 1 (EN1), Wilms' tumor 1 (WT1), fibroblast growth factor 1 (FGF1), and fibroblast growth factor receptor 2 (FGFR2). The gene expression of these cell differentiation markers in hASCs was normalized to control levels in patients with inactive disease. We also found genes related to cell differentiation that were downregulated in hASCs from patients with active disease, including the PR domain zinc finger protein 8 (PRDM8), PR domain zinc finger protein 16 (PRDM16), distal-less homeobox 5 (DLX5), and notch receptor 4 (NOTCH4). The gene expression of these cell differentiation markers in hASCs was not normalized to control levels in patients with inactive disease with the 


\section{A. Immune system \\ Control-ASCs $\square$ Active CD-ASCs Inactive CD-ASCs}
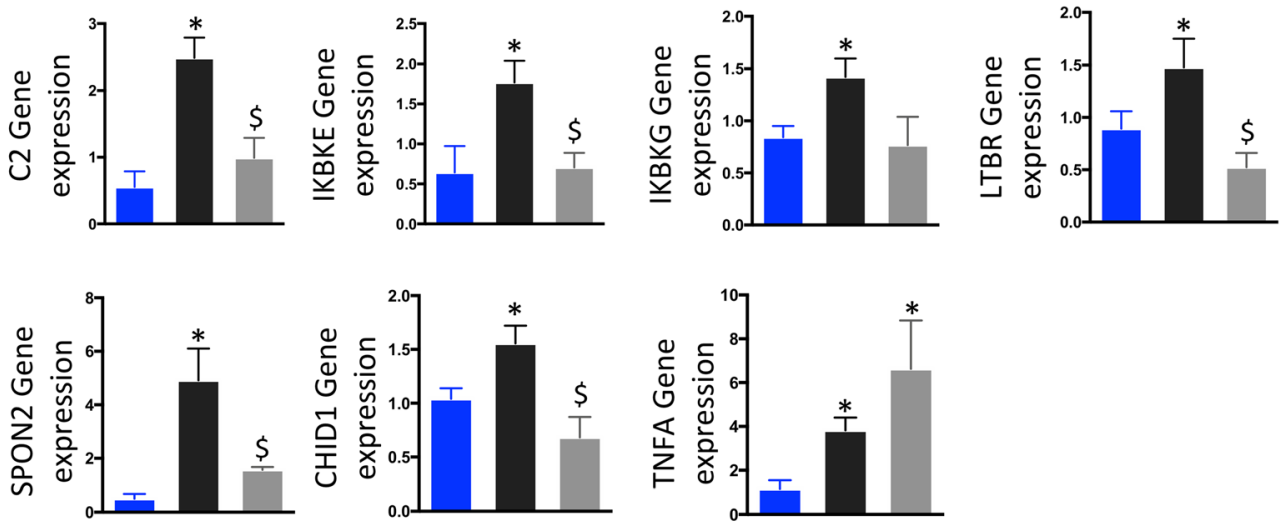

\section{B. Metabolic process}
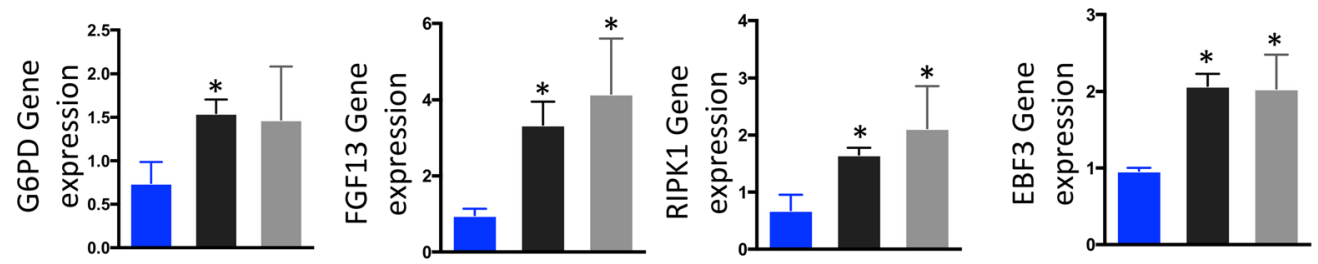

\section{Cell Differentiation}
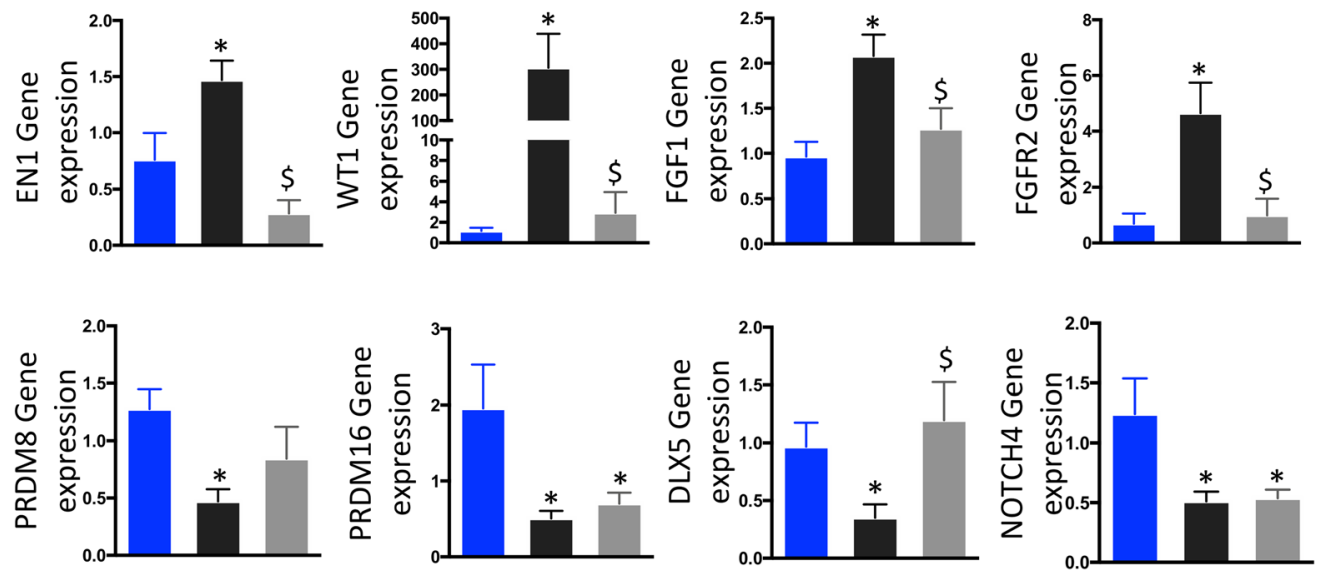

\section{Development process}
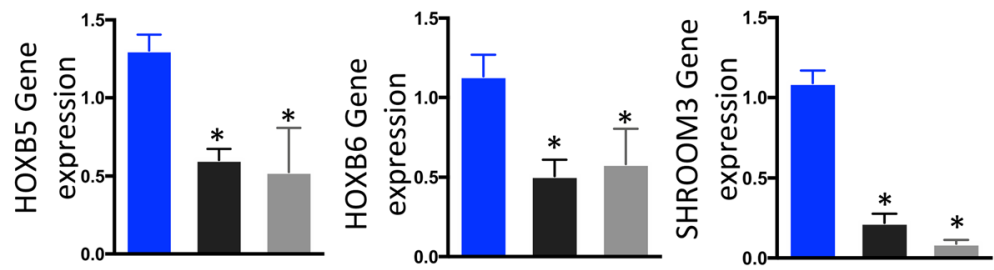

Fig. 4 (See legend on next page.) 
(See figure on previous page.)

Fig. 4 Gene expression of candidate genes obtained from differentially methylated regions between hASCs isolated from patients with active or inactive Crohn's disease and control individuals. a Genes involved in immune system response: C2, IKBKE, IKBKG, LTBR, SPON2, CHIDI, and TNFA. b Genes related to the regulation of metabolism: G6PD, FGF13, EBF3, and RIPK1. c Genes related to cell differentiation: EN1, WT1, FGF1, FGFR2, PRDM8, PRDM16, and DLX5. d Genes involved in development process: HOXB5, HOXB6 and SHROOM3. One-way analysis of variance with multiple comparisons corrected by Newman-Keuls test was used. ${ }^{*} p<0.05$ versus control-hASCs; ${ }^{\$} p<0.05$ versus active CD-hASCs

exception of $D L X 5$, a gene implicated in osteoblastic differentiation in mesenchymal stem cells [30].

\section{Development process}

We also studied the expression of genes related to development process, such as the homeobox B5 (HOXB5), homeobox B6 (HOXB6), and shroom family member 3 (SHROOM3), finding that they were all significantly downregulated in hASCs from patients with active or inactive disease compared with controls (Fig. 4d). This suggests that epigenetic modifications in these regions remain across $\mathrm{CD}$ progression.

\section{Reproducibility of the observed epigenetic changes in peripheral blood}

Lastly, we examined whether the specific epigenetic signatures in hASCs were reproduced in a more easily accessible sample from the patients' peripheral blood. Accordingly, we tested the expression of candidate genes in cohort III (PBMC cohort isolated from three groups: $n=10 / 10$ active/inactive $\mathrm{CD}$ and $n=10$ controls), to validate the changes in gene expression observed in hASCs between healthy controls and patients with CD. Figure 5 shows the expression data for the 9 genes in which we observed significant variability in hASCs. We found that the genes that showed the same behavior in PBMCs and hASCs were those related to immune system and cell differentiation. Accordingly, PBMC expression of the immune system-related genes C2, SPON2, and $L T B R$ was normalized in patients with inactive CD, whereas TNFA expression was not (Fig. 5a). Similarly, PBMC expression of cell differentiation-related genes EN1, WT1, and FGFR2 was at control levels in patients with inactive disease with the exception of PRDM16, which was not significantly recovered in these patients (Fig. 5b). No significant differences were found in gene expression for the other genes (IKBKE, IKBKG, CHID1, G6PD, RIPK1, FGF13, EBF3, FGF1, PRDM8, DLX5) (data not shown).

\section{Discussion}

We previously reported that hASCs isolated from the subcutaneous adipose tissue (SAT) of patients with CD mimic some of the features of equivalent cells from the $\mathrm{CF}$ of the affected colonic tissue, including functional defects [18]. Here, we report a specific epigenetic DNA methylation signature in hASCs from the SAT of patients with CD. Moreover, we show that changes to DNA methylation-related gene expression both in hASCs and PBMCs are only partially restored in patients with inactive $C D$, overall suggesting the persistence of latent epigenetic modifications involving several genes mainly related to immune system and metabolic and cell differentiation processes.

\section{Differentially methylated positions in patients with Crohn's disease}

The examination of the chromosomal distribution of DMPs in hASCs between patients with CD and healthy controls revealed no significant differences; however, our analysis yielded 17 associated genes with more than ten CpG sites significantly differentially methylated. The two most significant DMPs were found in PTPRN2 and PRMD16. PTPRN2 encodes a protein tyrosine phosphatase implicated in various biological processes including tumor pathogenesis and autoimmune disease [31-34]. Supporting our present findings, PTPRN2 has previously been reported to be the most significant DMP in PBMCs from patients with CD [19]. Interestingly, PTPRN2 shows dysregulated DNA methylation in obese children [31], pointing to a link between metabolic dysfunction and immune response. Indeed, specific protein tyrosine phosphatases associated with autoimmune diseases have been detected in rat gastrointestinal endocrine cells [35]. Our finding of a dysregulation of DNA methylation in PTPRN2 in patients with CD (Fig. 2e, Supplementary Figure S4) suggests that this region could be a hot-spot risk marker that deserves further study. This change in DNA methylation was, nevertheless, not reflected by significant changes in gene expression (although there was a trend for downregulation) (Supplementary Figure S4), possibly because of the small sample size used. PRMD16 is a transcription factor that activates brown fatspecific genes and is also associated with insulin sensitivity in obese subjects, linking adipose tissue expression and systemic metabolic homeostasis [36]. The gene expression analysis revealed significantly lower expression of PRDM16 both in hASCs and PBMCs from patients relative to controls. The downregulation of PRDM16 and PTPNR2 expression was independent of the clinical status of $C D$, suggesting a persistence of the epigenetic changes in patients with $\mathrm{CD}$ despite a diagnosis of clinical remission. Overall, it is tempting to speculate on a putative link between $\mathrm{CD}$ and dysmetabolism mediated by AT. 


\section{A. Immune system}
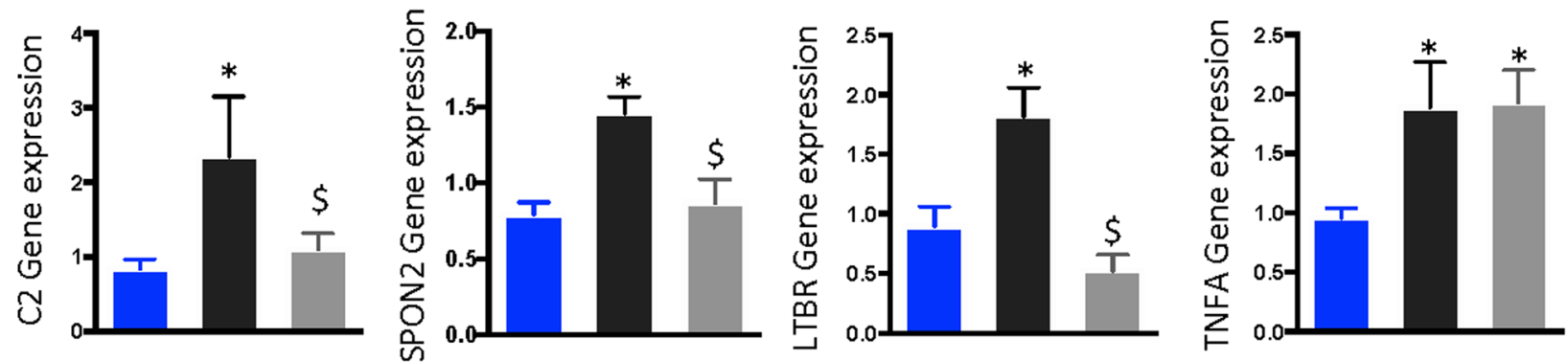

\section{B. Cell Diferentiation}
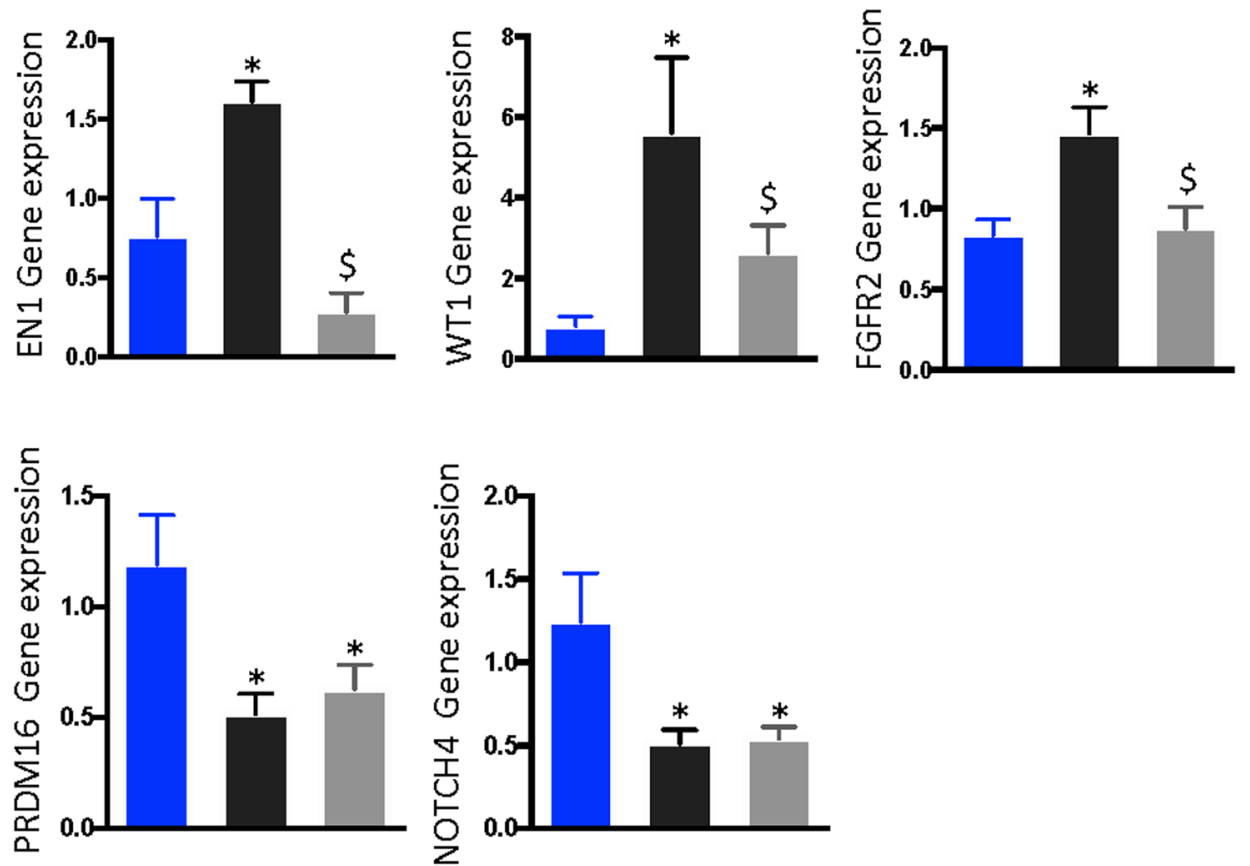

Fig. 5 Gene expression of candidate genes obtained from differentially methylated regions between PBMCs isolated from patients with active or inactive Crohn's disease and control individuals. a Genes involved in immune system response: C2, SPON2, LTBR, and TNFA. b Genes related to cell differentiation: EN1, WT1, FGFR2, and PRDM16. One-way analysis of variance with multiple comparisons corrected by Newman-Keuls test was used. ${ }^{*} p<0.05$ versus control-PBMCs; $\$ p<0.05$ versus active CD-PBMCs

\section{Differentially methylated regions in Crohn's disease}

The analysis of DMRs in hASCs identified four major categories: immune system, metabolic, cell differentiation, and development processes. Examination of the expression of the most representative genes in these categories highlighted a concurrence with the methylation data in patients from the discovery cohort I. Accordingly, we confirmed an increase in pro-inflammatory markers such as SPON2, which is highly expressed both in colon and in adipose tissue and promotes M1-like macrophage recruitment [37]; $C 2$, which activates complement and triggers inflammation [38]; IKBKE and $I K B K B$, key drivers of the NF-kappa-B inflammatory pathway [39]; $C H 1 D 1$, which has been recently reported to have high expression in PBMCs from patients with rheumatoid arthritis [40], functioning as a regulator of the inflammatory response by macrophages; and TNFA, a gene involved in the regulation of a wide spectrum of biological processes and implicated in a variety of diseases, including autoimmune disease and insulin resistance, among others [41-43]. The gene expression of the tested immune system genes was normalized in hASCs of patients in disease remission with the exception of TNFA, which remained highly expressed even in the inactive period of $C D$. These results are in agreement with our previous study showing that TNFA gene expression in hASCs remains high during CD relapse 
[18] and suggest that epigenetic modifications might induce the persistence of a latent inflammation in a background of apparent clinical remission.

Genes related to different metabolic process were also modified. We found a significant increase in the expression of genes involved in several metabolic intra-cellular pathways (enzymes, kinases, and growth factors, etc.) in hASCs from patients with active disease, and high gene expression levels were also preserved during the inactive disease period, indicating a persistence of metabolic disturbances.

Regarding markers of cell differentiation processes in hASCs, we observed a dual expression pattern: an increase in genes mostly related to adipogenesis and/or osteoblast differentiation (EN1,WT1, FGF1, and FGFR2) and a decrease in transcription factors relevant to cell differentiation and maturation (PRDM8, PRDM16, and $D L X 5)$ with a return to healthy control levels during $C D$ remission (with the exception of PRDM16, as described above).

Finally, genes involved in development processes were downregulated in CD-hASCs. Again, disease remission was not accompanied by the restoration of mRNA expression of the studied genes. HOX genes are among the best studied genes in developmental biology and are responsible for driving the differentiation of tissue stem cells along their respective lineages to maintain the correct function of tissues and organs [44]. Thus, downregulation of these genes might indicate an alteration in the stemness function of hASCs in patients with $\mathrm{CD}$, which could help to explain some of the functional alterations (loss of anti-inflammatory and regenerative capacity) previously described [18].

\section{Verification of candidate genes in circulating PBMCs in patients with Crohn's disease}

Modifications in the gene expression profile observed in hASCs according to the methylation pattern were also explored in PBMCs, as they represent a more accessible source for sampling. Also, epigenetic modifications to hASCs might be reflected in peripheral blood, as already demonstrated in other inflammatory diseases such as obesity [45]. Indeed, changes to the PBMC methylome in $\mathrm{CD}$ have previously been described, revealing that differentially methylated genes implicated in immune response are the most frequently affected $[19,46]$. In agreement with these reports, we found the same enriched GO terms in hASCs in CD such as immune response and immune system process, and we found that some of the changes found in hASCs were replicated in PBMCs, even when patients were classified according to clinical status.

Globally, we found that alterations in DNA methylation of hASCs in CD patients affect mainly immune system, cell differentiation, metabolic, and development processes and that changes to DNA methylation correlate with changes in gene expression. Some of these alterations were maintained despite the clinical remission of the disease. These observations may have important repercussions in the clinical setting, in particular the use of hASCs for autologous transplantation in some complications of $\mathrm{CD}$, considering their immune-suppressive and regenerative capabilities [47-49].

This study has some limitations that warrant discussion. It is an observational study, with a limited number of cases, and it would be necessary to monitor the evolution of the patients to evaluate the repercussions of the methylation changes for the natural history of the disease. Larger prospective studies in patients will be necessary to decipher the clinical relevance of these epigenetic changes.

Overall, our study has several strengths. First, we present a specific methylome signature in adipocyte precursors from the SAT of patients with CD, including significant DMRs. Second, we show that these epigenetic changes translate into apparent changes in the mRNA expression of several genes, with a special bearing on inflammatory pathways, which are only partially reversed during remission periods of the disease. Finally, we demonstrate that these changes are replicated in PBMCs, pointing to a subtle persistence of the epigenetic changes observed in active disease despite a clinical diagnosis of remission.

\section{Conclusions}

Adipose-stem cells isolated from patients with $\mathrm{CD}$ exhibit altered DNA methylation and gene expression profiles, with immune system, cell differentiation, metabolic, and development processes identified as the main pathways affected. Interestingly, the gene expression of several genes involved in these pathways is only partially restored in patients with inactive $\mathrm{CD}$, both in hASCs and PBMCs. Understanding how $\mathrm{CD}$ shapes the functionality of hASCs is critical for investigating the complex pathophysiology of this disease, as well as for the success of cell-based therapies.

\section{Methods}

\section{Study population}

Patients and controls were recruited at the University Hospital Joan XXIII (Tarragona, Spain) and University Hospital Vall d'Hebrón (Barcelona, Spain) in accordance with the tenets of the Declaration of Helsinki. The corresponding hospital ethics committees approved the study and written informed consent was obtained from all participants before entering the study. Donors were classified as those in relapse (active) or in remission (inactive) according to the CDAI score as well as biological and 
clinical parameters such as PCR and fecal calprotectin. Furthermore, endoscopic evaluation was performed in the majority of patients $[20,21,50,51]$. Healthy subjects and donors with inactive $\mathrm{CD}$ were recruited from ageand gender-matched subjects undergoing non-acute surgical interventions such as hernia or cholecystectomy, in a scheduled routine surgery. Patients with active CD were recruited from those undergoing surgery for symptomatic complications. The following three cohorts were included in the study: cohort I-methylation study. hASCs were isolated from SAT biopsies of 7 patients with active $\mathrm{CD}$ and 5 healthy controls; $71 \%$ of patients had endoscopic evaluation. Cohort II-gene expression study. The number of subjects included in the first cohort was increased to 10. Accordingly, hASCs were isolated from SAT biopsies of 10 active and 10 inactive patients with $\mathrm{CD}$ and 10 healthy controls. Of note, the correlation studies between methylation and gene expression data were only performed between data from the same subject; $80 \%$ of CD patients had endoscopic evaluation. Cohort III-PBMC study cohort. Thirty new subjects were enrolled in this cohort. PBMCs were isolated from 20 patients with CD (10 active and 10 inactive) and 10 healthy controls. Biochemical and anthropometric variables of cohorts I, II, and II are presented in Supplementary Tables S1, S2, and S3, respectively.

\section{Adipose stem cell isolation and culture}

hASCs were isolated as described [52, 53]. Briefly, SAT was washed extensively with phosphate buffered saline (PBS) to remove debris and then treated with $0.1 \%$ collagenase in PBS-1\% BSA for $1 \mathrm{~h}$ at $37^{\circ} \mathrm{C}$ with gentle agitation. Digested samples were centrifuged at $300 \times g$ at $4{ }^{\circ} \mathrm{C}$ for $5 \mathrm{~min}$ to separate adipocytes from stromal cells. The cell pellet containing the stromal fraction was resuspended in stromal culture medium consisting of DMEM/F12 medium, 10\% fetal bovine serum, and 1\% antibiotic/antimycotic solution. To prevent spontaneous differentiation, primary cultures of hASCs at passage 0 (P0) were grown to $90 \%$ confluence and harvested with trypsin-EDTA, and then aliquots $\left(1 \times 10^{6}\right.$ cells $)$ were cryopreserved in liquid nitrogen until required [54].

\section{Adipose stem cell immunophenotyping}

Cells $\left(2 \times 10^{5}\right)$ were incubated with a panel of primary antibodies (described in Supplementary Table S4) [16]. After isolation, the minimal functional and quantitative criteria, established by the International Society of Cell Therapy and the International Federation for Adipose Therapeutics and Science, were confirmed by flow cytometry $[16,18]$. All experiments were performed in cells at P3 or P4.
DNA methylation profiling using universal bead array Genomic DNA was extracted from cells using the NucleoSpin ${ }^{\oplus}$ Tissue Kit (Macherery-Nagel GmbH, Dueren, Germany). DNA (cytosine) methylation profiles were generated by combining bisulfite conversion of genomic DNA and whole-genome amplification with direct, array-based capture and scoring of the CpG (cyotosine-guanine) loci. DNA samples were processed and hybridized to the Illumina EPIC array or the 850k Infinium Human-MethylationEPIC BeadChip (Illumina Inc., San Diego, CA), developed to quantitatively assay more than 850,000 methylation sites across the genome at single-nucleotide resolution, following the Infinium HD Methylation Assay Protocol. Hybridized BeadChips were imaged on an Illumina iScan system following the manufacturer's recommendations. Gene annotation was performed using Illumina's annotation of probes. Briefly, CpG markers present on MethylationEPIC 850k array were classified based on their chromosome location, the Infinium chemistry used to interrogate the marker (Infinium I, Infinium II), and the feature category gene region as per UCSC annotation (TSS200, TSS1500, 5' UTR, 1st Exon, Body, 3' UTR) [55]. Methylation data has been deposited in the GEO database with the accession code GSE138311.

\section{Differentially methylated loci analysis}

All statistical data treatments were performed with the $R$ statistical programming environment (version 3.5.3; $\mathrm{R}$ core team, 2019). DNA methylation raw data (IDAT files, Illumina iScan system direct output storing probe intensities) were read using the Bioconductor package minfi (version 1.28.3) $[56,57]$ and the annotation packages IlluminaHumanMethylationEPICmanifest version 3.0 and IlluminaHumanMethylationEPICanno.ilm10b2.hg19 version 6.0. Initial pre-processing and quality control were performed with minfi. The quality of each sample was assessed using the internal control probes located on the BeadChip array and samples with a $p$ value $>0.01$ were discarded. Probe intensities were normalized using the Stratified Quantile Normalization method [58], based on the assumption that probes with the same CpG annotation should have similar distributions. A total of $9.32 \%$ of the probes were removed as they were identified as failed in one or more samples, mapped to multiple genome sites, or associated with CpGs with known single-nucleotide polymorphisms. The $\mathrm{CpG}$ methylation level was calculated as $M$ values $\left(M=\log _{2}[M / U]\right)$, used for statistical analyses, and $\beta$ values $(\beta=M /[M+U+100])$, used for methylation level visualization [59]. DMPs were located through linear regression using dmpFinder, a minfi wraparound lmFit function of the limma software package [60].

Based on Illumina's manifest, DMPs were assigned to genes and candidate genes were identified based on a 
nominal $p$ value cutoff $<1 \times 10^{-4}$ and ten or more DMPs per gene. DMRs were located using the minfi function Bumphunter [56], originally described by Jaffe et al. [29], with default settings and the smoothing option. In brief, Bumphunter looks for DMRs by searching for CpGs with a mean difference above a certain threshold. We set the inclusion threshold to 0.2. To remove non-biologically significant DMRs, we filtered for at least three consecutive CpGs. Again, significant DMRs were assigned to genes based on Illumina's manifest, and candidate genes were selected based on $p$ value cutoff $<1 \times 10^{-2}$ and the number of DMRs per gene and their relation to the gene structure.

\section{Functional network analysis and visualization}

For functional studies, STRING v11: protein-protein association networks (https://string-db.org/) was used to evaluate the implicated molecular and functional pathways for the candidate genes. We corroborated these enriched pathways using REVIGO (http://revigo.irb.hr/), which summarizes and visualizes lists of GO terms. Specifically, REVIGO was used to cluster significant enriched GO terms $(F D R<0.05)$ into similar functions.

\section{Isolation of human peripheral blood mononuclear cells}

Human PBMCs were isolated with Ficoll-Hypaque gradients (Amersham Bioscience, Barcelona. Spain). PBMC pellets were frozen and stored at $-80^{\circ} \mathrm{C}$ until DNA extraction.

\section{Gene expression analysis}

Total RNA was isolated using the RNeasy Lipid Tissue Mini Kit (Qiagen, Hilden, Germany). RNA quantity was measured at $260 \mathrm{~nm}$ and purity was assessed by the OD260/OD280 ratio. One microgram of RNA was transcribed to cDNA with random primers using the Reverse Transcription System (Applied Biosystems, Foster City, CA). Quantitative gene expression was evaluated by realtime quantitative PCR (qPCR) on a 7900HT Fast RealTime PCR System using the TaqMan Gene Expression Assay (Applied Biosystems), listed in Supplementary Table S5. Gene expression values were calculated using the comparative $\mathrm{Ct}$ method and expressed relative to the expression of the housekeeping gene 18S (Hs03928985_g1).

\section{Statistical analysis}

For gene expression data, experimental results were presented as mean \pm standard deviation (SD) from 10 independent experiments (independent donors), performed at least in duplicate. Comparisons between three groups were performed using nonparametric analysis of variance for multiple comparisons and Newman-Keuls correction (significance, $p<0.05$ ). Statistical calculations and visualizations were performed using GraphPad Prism 6 (GraphPad Software Inc., San Diego, CA).

For clinical and anthropometrical variables, normally distributed data were expressed as mean $\pm \mathrm{SD}$, and for variables with no Gaussian distribution, values were expressed as median (interquartile range). Statistical analysis was performed with the Statistical Package for the Social Sciences software, version 15 (SPSS, Chicago, IL).

\section{Supplementary information}

Supplementary information accompanies this paper at https://doi.org/10. 1186/s13148-020-00843-3.

Additional file 1. Supplementary file 1.

Additional file 2. Supplementary file 2.

Additional file 3. Figure S1-S4, Table S1-S5.

\section{Abbreviations}

CD: Crohn's disease; CF: Creeping fat; hASCs: Human adipose-derived stem cells; PBMCs: Peripheral blood mononuclear cells; IBD: Inflammatory bowel diseases; AT: Adipose tissue; SAT: Subcutaneous adipose tissue; PCA: Principal component analysis; BMI: Body mass index; FMRs: Fully methylated regions; LMRs: Low methylated regions; UMRs: Unmethylated regions;

DMPs: Differentially methylated positions; DMR: Differentially methylated regions; GO: Gene ontology; TSS: Transcription start site; UTR: 5' untranslated region; ExonBnd: Exon boundary; FDR: False discovery rate

\section{Acknowledgements}

We want to particularly acknowledge the patients and the BioBank IISPV (PT17/0015/0029) integrated in the Spanish National Biobanks Network for its collaboration. We thank Dr. Kenneth McCreath for helpful comments on the manuscript.

\section{Author contributions}

CS, ME, MT-P, CN-R, and DM-F carried out the experiments and generated data. CS and AS-V performed the epigenetics analysis. MMillan, MB, MMenacho, MMarti, and EE carried out part of the study population selection and human sample processing. CS, MMillan, ME, JV, and SF-V contributed to the discussion and reviewed the manuscript. CS, JV, and SF-V conceived the study, discussed the data, and wrote the manuscript. JV and SF-V are the guarantors of this work. The author(s) read and approved the final manuscript.

\section{Funding}

This study was supported by grants from the Spanish Ministry of Economy and Competitiveness [PI18/00037, PI15/00143 to CS; PI14/00228 and PI17/ 01503 to J.V., SAF2015-65019-R and RTI2018-093919 to S.F.-V], co-financed by the European Regional Development Fund (ERDF), and a European Crohn's and Colitis Organization (ECCO) grant to CS. The Spanish Biomedical Research Center in Diabetes and Associated Metabolic Disorders (CIBERDEM) [CB07708/0012] is an initiative of the Instituto de Salud Carlos III. CS acknowledges support from the "Ramón y Cajal" program from the Ministerio de Educación y Ciencia [RYC2013-13186], co-financed by ERDF. SF-V acknowledges support from the "Miguel Servet" tenure track program [CP10/00438 and CPII16/0008] from the Fondo de Investigación Sanitaria, co-financed by the ERDF. ME is recipient of a Generalitat de Catalunya grant for the incorporation of scientists PERIS [SLT002/16/00120], and M.T-P is recipient of Río Hortega IISCIII fellowship [CM18/00029], both co-financed by the ERDF.

Availability of data and materials

Authors consent to the availability of data and materials.

Ethics approval and consent to participate

The study has been carried out in accordance with the guidelines of Declaration of Helsinki, and the corresponding hospital ethics committees 
approved the study; written informed consent was obtained from all participants before entering the study.

\section{Consent for publication}

All the authors approved the manuscript and consented for publication.

\section{Competing interests}

The authors declare that they have no competing interests.

\section{Author details}

'Institut d'Investigació Sanitària Pere Virgili, Hospital Universitari Joan XXIII, Dr Mallafré Guasch, 4, 43007 Tarragona, Spain. ${ }^{2}$ CIBER de Diabetes y Enfermedades Metabólicas Asociadas (CIBERDEM), Instituto de Salud Carlos III, 28014, Madrid, Spain. ${ }^{3}$ Colorectal Surgery Unit, Hospital Universitari Joan XXIII, 43007 Tarragona, Spain. ${ }^{4}$ Colorectal Surgery Unit, Hospital Universitari La Fe, Valencia, Spain. ${ }^{5}$ Digestive Unit, Hospital Universitari Joan XXIII, 43007 Tarragona, Spain. ${ }^{6}$ Colorectal Surgery Unit, General Surgery Service, Hospital Valle de Hebron, Universitat Autonoma de Barcelona, 08035 Barcelona, Spain. ${ }^{7}$ Universitat Rovira i Virgili, Tarragona, Spain.

Received: 13 December 2019 Accepted: 24 March 2020

Published online: 06 April 2020

\section{References}

1. Kaplan GG. The global burden of IBD: from 2015 to 2025. Nat. Rev. Gastroenterol. Hepatol. 2015;12:720-7.

2. Alatab S, Sepanlou SG, Ikuta K, Vahedi H, Bisignano C, Safiri S, et al. The global, regional, and national burden of inflammatory bowel disease in 195 countries and territories, 1990-2017: a systematic analysis for the Global Burden of Disease Study 2017. Lancet Gastroenterol Hepatol. 2020;5:17-30.

3. Büning C, Von Kraft C, Hermsdorf M, Gentz E, Wirth EK, Valentini L, et al. Visceral adipose tissue in patients with Crohn's disease correlates with disease activity, inflammatory markers, and outcome. Inflamm Bowel Dis. 2015;21:2590-7.

4. Connelly TM, Juza RM, Sangster W, Sehgal R, Tappouni RF, Messaris E. Volumetric fat ratio and not body mass index is predictive of ileocolectomy outcomes in Crohn's disease patients. Dig Surg. 2014;31:219-24.

5. Fink C, Karagiannides I, Bakirtzi K, Pothoulakis C. Adipose tissue and inflammatory bowel disease pathogenesis. Inflamm. Bowel Dis. 2012;18: 1550-7.

6. Li Y, Zhu W, Gong J, Zhang W, Gu L, Guo Z, et al. Visceral fat area is associated with a high risk for early postoperative recurrence in Crohn's disease. Color Dis. 2015;17:225-34.

7. Coffey CJ, Kiernan MG, Sahebally SM, Jarrar A, Burke JP, Kiely PA, et al. Inclusion of the mesentery in ileocolic resection for Crohn's disease is associated with reduced surgical recurrence. J Crohn's Colitis. 2018;12:1139-50.

8. Kane $H$, Lynch L. Innate immune control of adipose tissue homeostasis. Trends Immunol. 2019:40:857-72.

9. Francisco V, Pino J, Gonzalez-Gay MA, Mera A, Lago F, Gómez R, et al. Adipokines and inflammation: is it a question of weight? $\mathrm{Br} J$ Pharmacol. 2018;175:1569-79.

10. Zmora N, Bashiardes S, Levy M, Elinav E. The role of the immune system in metabolic health and disease. Cell Metab. Elsevier. 2017;25:506-21.

11. Grant RW, Dixit VD. Adipose tissue as an immunological organ. Obesity. Blackwell Publishing Inc.; 2015;23:512-8.

12. Strong AL, Burow ME, Gimble JM, Bunnell BA. Concise review: the obesity cancer paradigm: Exploration of the interactions and crosstalk with adipose stem cells. Stem Cells. 2015:33:318-26.

13. Aranda P, Agirre X, Ballestar E, Andreu EJ, Román-Gómez J, Prieto I, et al. Epigenetic signatures associated with different levels of differentiation potential in human stem cells. PLoS One. 2009;4:e7809.

14. Fadini GP, Bonora BM, Marcuzzo G, Marescotti MC, Cappellari R, Pantano G, et al. Circulating stem cells associate with adiposity and future metabolic deterioration in healthy subjects. J Clin Endocrinol Metab. 2015;100:4570-8.

15. He Q, Wan C, Li G. Concise Review: Multipotent mesenchymal stromal cells in blood. Stem Cells. 2007;25:69-77.

16. Pachón-Peña G, Serena C, Ejarque M, Petriz J, Duran X, Oliva-Olivera W, et al. Obesity determines the immunophenotypic profile and functional characteristics of human mesenchymal stem cells from adipose tissue. Stem Cells Transl Med. 2016;5:464-75.
17. Serena C, Keiran N, Ceperuelo-Mallafre V, Ejarque M, Fradera R, Roche K, et al. Obesity and Type 2 Diabetes Alters the Immune Properties of Human Adipose Derived Stem Cells. Stem Cells. 2016;34:2559-73.

18. Serena C, Keiran N, Madeira A, Maymó-Masip E, Ejarque M, Terrón-Puig M, et al. Crohn's disease disturbs the immune properties of human adiposederived stem cells related to inflammasome activation. Stem Cell Reports. 2017:9:1109-23.

19. Li Yim AYF, Duijvis NW, Zhao J, de Jonge WJ, D'Haens GRAM, Mannens MMAM, et al. Peripheral blood methylation profiling of female Crohn's disease patients. Clin Epigenetics [Internet]. Clin Epigenetics; 2016;8:1-13. Available from: https://doi.org/10.1186/s13148-016-0230-5.

20. Somineni HK, Venkateswaran S, Kilaru V, Marigorta UM, Mo A, Okou DT, et al. Blood-derived DNA methylation signatures of Crohn's Disease and severity of intestinal inflammation. Gastroenterology. 2019;156:2254-2265.e3.

21. Moret-Tatay I, Cerrillo E, Sáez-González E, Hervás D, Iborra M, Sandoval J et al. Identification of epigenetic methylation signatures with clinical value in Crohn's disease. Clin Transl Gastroenterol. 2019;10:e00083.

22. Stadler MB, Murr R, Burger L, Ivanek R, Lienert F, Schöler A, et al. DNAbinding factors shape the mouse methylome at distal regulatory regions. Nature. 2011;480:490-5.

23. Ejarque M, Ceperuelo-Mallafré V, Serena C, Maymo-Masip E, Duran X, DíazRamos A, et al. Adipose tissue mitochondrial dysfunction in human obesity is linked to a specific DNA methylation signature in adipose-derived stem cells. Int J Obes [Internet]. Springer US; 2019;43:1256-68. Available from: https://doi.org/10.1038/s41366-018-0219-6.

24. Sheaffer KL, Kim R, Aoki R, Elliott EN, Schug J, Burger L, et al. DNA methylation is required for the control of stem cell differentiation in the small intestine. Genes Dev. 2014:28:652-64.

25. Durek P, Nordström K, Gasparoni G, Salhab A, Kressler C, de Almeida M, et al. Epigenomic profiling of human CD4+ T cells supports a linear differentiation model and highlights molecular regulators of memory development. Immunity. 2016:45:1148-61.

26. Manzo M, Wirz J, Ambrosi C, Villaseñor R, Roschitzki B, Baubec T. Isoformspecific localization of DNMT3A regulates DNA methylation fidelity at bivalent CpG islands. EMBO J. 2017;36:3421-34.

27. Ventham NT, Kennedy NA, Adams AT, Kalla R, Heath S, O'Leary KR, et al. Integrative epigenome-wide analysis demonstrates that DNA methylation may mediate genetic risk in inflammatory bowel disease. Nat Commun. 2016;7:13507.

28. Ball MP, Li JB, Gao Y, Lee J-H, Leproust E, Park I-H, et al. Targeted and genome-scale methylomics reveals gene body signatures in human cell lines. Nat Biotechnol. 2009:27:361-8.

29. Jaffe $A E$, Murakami $P$, Lee $H$, Leek JT, Fallin MD, Feinberg AP, et al. Bump hunting to identify differentially methylated regions in epigenetic epidemiology studies. Int J Epidemiol. 2012;41:200-9.

30. Pino AM, Rosen CJ, Pablo RJ. In Osteoporosis, differentiation of mesenchymal stem cells (MSCs) improves bone marrow adipogenesis. Biol Res. 2012:45:279-87.

31. Lee S. The association of genetically controlled CpG methylation (cg158269415) of protein tyrosine phosphatase, receptor type N2 (PTPRN2) with childhood obesity. Sci Rep. 2019;9:1-7.

32. Sengelaub CA, Navrazhina K, Ross JB, Halberg N, Tavazoie SF. PTPRN 2 and PLC $\beta 1$ promote metastatic breast cancer cell migration through PI $(4,5) \mathrm{P} 2$ -dependent actin remodeling. EMBO J. 2016;35:62-76.

33. Chen PY, Chu A, Liao WW, Rubbi L, Janzen C, Hsu FM, et al. Prenatal Growth Patterns and Birthweight Are Associated With Differential DNA methylation and gene expression of cardiometabolic risk genes in human placentas: a discovery-based approach. Reprod Sci. 2018;25:523-39.

34. Coit P, Ognenovski M, Gensterblum E, Maksimowicz-Mckinnon K, Wren $J D$, Sawalha AH. Ethnicity-specific epigenetic variation in naïve CD4+ cells and the susceptibility to autoimmunity. Epigenetics Chromatin. 2015:8:1-13.

35. Gomi H, Kubota-Murata C, Yasui T, Tsukise A, Torii S. Immunohistochemical Analysis of IA-2 Family of Protein Tyrosine phosphatases in rat gastrointestinal endocrine cells. J Histochem Cytochem. 2013;61:156-68.

36. Moreno-Navarrete JM, Ortega F, Moreno M, Xifra G, Ricart W, FernándezReal JM. PRDM16 sustains white fat gene expression profile in human adipocytes in direct relation with insulin action. Mol Cell Endocrinol. 2015; 405:84-93.

37. Zhang YL, Li Q, Yang XM, Fang F, Li J, Wang YH, et al. Spon2 promotes $\mathrm{m} 1$ like macrophage recruitment and inhibits hepatocellular carcinoma 
metastasis by distinct integrin-rho gtpase-hippo pathways. Cancer Res. 2018:78:2305-17.

38. Markiewski MM, Lambris JD. The role of complement in inflammatory diseases from behind the scenes into the spotlight. Am J Pathol. 2007;171:715-27.

39. Yamamoto Y, Gaynor RB. IkB kinases: key regulators of the NF-kB pathway. Trends Biochem Sci. 2004;29:72-9.

40. Xiao W, Meng G, Zhao Y, Yuan H, Li T, Peng Y, et al. Human secreted stabilin-1-interacting chitinase-like protein aggravates the inflammation associated with rheumatoid arthritis and is a potential macrophage inflammatory regulator in rodents. Arthritis Rheumatol. 2014:66:1141-52.

41. Kunz M, Ibrahim SM. Cytokines and cytokine profiles in human autoimmune diseases and animal models of autoimmunity. Mediators Inflamm. 2009; 2009:979258.

42. Gibson PR. Increased gut permeability in Crohn's disease: Is TNF the link? Gut. 2004;53:1724-5.

43. Valenti L, Fracanzani AL, Dongiovanni P, Santorelli G, Branchi A, Fiorelli G, et al. Tumor necrosis factor a promoter polymorphisms and insulin resistance in nonalcoholic fatty liver disease. Gastroenterology. 2002;122: 274-80.

44. Seifert A. Role of Hox genes in stem cell differentiation. World J Stem Cells [Internet]. 2015;7:583. Available from: http://www.wjgnet.com/1948-0210/ full/v7/i3/583.htm.

45. Crujeiras AB, Diaz-Lagares A, Sandoval J, Milagro Fl, Navas-Carretero S, Carreira MC, et al. DNA methylation map in circulating leukocytes mirrors subcutaneous adipose tissue methylation pattern: a genome-wide analysis from non-obese and obese patients. Sci Rep [Internet]. Nature Publishing Group; 2017;7:1-13. Available from: https://doi.org/10.1038/srep41903.

46. McDermott E, Ryan EJ, Tosetto M, Gibson D, Burrage J, Keegan D, et al. DNA methylation profiling in inflammatory bowel disease provides new insights into disease pathogenesis. J Crohns Colitis. 2016;10:77-86.

47. Panés J, García-Olmo D, Van Assche G, Colombel JF, Reinisch W, Baumgart DC, et al. Expanded allogeneic adipose-derived mesenchymal stem cells (Cx601) for complex perianal fistulas in Crohn's disease: a phase 3 randomised, double-blind controlled trial. Lancet. 2016;388:1281-90.

48. Dietz AB, Dozois EJ, Fletcher JG, Butler GW, Radel D, Lightner AL, et al. Autologous mesenchymal stem cells, applied in a bioabsorbable matrix, for treatment of perianal fistulas in patients with Crohn's disease. Gastroenterology. 2017;153:59-62.e2.

49. Lee WY, Park KJ, Cho YB, Yoon SN, Song KH, Kim DS, et al. Autologous adipose tissue-derived stem cells treatment demonstrated favorable and sustainable therapeutic effect for Crohn's fistula. Stem Cells. 2013;31:2575-81.

50. Best WR, Becktel JM, Singleton JW, Kern F. Development of a Crohn's disease activity index. Gastroenterology. 1976;70:439-44.

51. Van Assche G, Dignass A, Reinisch W, van der Woude CJ, Sturm A, De Vos $\mathrm{M}$, et al. The second European evidence-based consensus on the diagnosis and management of Crohn's disease: Special situations. J. Crohn's Colitis. 2010:4:63-101.

52. Dubois SG, Floyd EZ, Zvonic S, Kilroy G, Wu X, Carling S, et al. Isolation of human adipose-derived stem cells from biopsies and liposuction specimens. Methods Mol Biol. 2008. https://doi.org/10.1007/978-1-60327-169-1_5.

53. Gimble JM, Guilak F. Adipose-derived adult stem cells: isolation, characterization, and differentiation potential. Cytotherapy [Internet]. Elsevier; 2003 [cited 2019 Jul 31];5:362-9. Available from: https://www. sciencedirect.com/science/article/abs/pii/S1465324903711104.

54. Pachón-Peña G, Yu G, Tucker A, Wu X, Vendrell J, Bunnell BA, et al. Stromal stem cells from adipose tissue and bone marrow of age-matched female donors display distinct immunophenotypic profiles. J Cell Physiol. 2011;226: 843-51.

55. Moran $\mathrm{S}$, Arribas $\mathrm{C}$, Esteller M. Validation of a DNA methylation microarray for $850,000 \mathrm{CpG}$ sites of the human genome enriched in enhancer sequences. Epigenomics. 2016;8:389-99.

56. Aryee MJ, Jaffe AE, Corrada-Bravo H, Ladd-Acosta C, Feinberg AP, Hansen $K D$, et al. Minfi: a flexible and comprehensive Bioconductor package for the analysis of Infinium DNA methylation microarrays. Bioinformatics. 2014;30: 1363-9.

57. Fortin JP, Triche TJ, Hansen KD. Preprocessing, normalization and integration of the Illumina HumanMethylationEPIC array with minfi. Bioinformatics. 2017;33:558-60.

58. Touleimat N, Tost J. Complete pipeline for Infinium ${ }^{\bullet}$ Human Methylation $450 \mathrm{~K}$ BeadChip data processing using subset quantile normalization for accurate DNA methylation estimation. Epigenomics. 2012;4:325-41.
59. Du P, Zhang X, Huang CC, Jafari N, Kibbe WA, Hou L, et al. Comparison of beta-value and M-value methods for quantifying methylation levels by microarray analysis. BMC Bioinformatics. 2010;11:587.

60. Ritchie ME, Phipson B, Wu D, Hu Y, Law CW, Shi W, et al. Limma powers differential expression analyses for RNA-sequencing and microarray studies. Nucleic Acids Res. 2015;43:e47.

\section{Publisher's Note}

Springer Nature remains neutral with regard to jurisdictional claims in published maps and institutional affiliations.

\section{Ready to submit your research? Choose BMC and benefit from:}

- fast, convenient online submission

- thorough peer review by experienced researchers in your field

- rapid publication on acceptance

- support for research data, including large and complex data types

- gold Open Access which fosters wider collaboration and increased citations

- maximum visibility for your research: over $100 \mathrm{M}$ website views per year

At BMC, research is always in progress.

Learn more biomedcentral.com/submissions 